\title{
Platelet-Derived Growth Factors in Non-GIST Soft-Tissue Sarcomas Identify a Subgroup of Patients with Wide Resection Margins and Poor Disease-Specific Survival
}

\author{
Thomas Karsten Kilvaer, ${ }^{1}$ Andrej Valkov, ${ }^{1,2}$ Sveinung W. Sorbye, ${ }^{1,2}$ Tom Donnem, ${ }^{3,4}$ \\ Eivind Smeland, ${ }^{4}$ Roy Martin Bremnes, ${ }^{3,4}$ and Lill-Tove Busund ${ }^{1,2}$ \\ ${ }^{1}$ Institute of Medical Biology, University of Tromsø, 9037 Troms $\varnothing$, Norway \\ ${ }^{2}$ Department of Clinical Pathology, University Hospital of North Norway, 9038 Tromsø, Norway \\ ${ }^{3}$ Institute of Clinical Medicine, University of Tromsø, 9037 Tromsø, Norway \\ ${ }^{4}$ Department of Oncology, University Hospital of North Norway, 9038 Troms $\varnothing$, Norway
}

Correspondence should be addressed to Thomas Karsten Kilvaer, kilvaer@gmail.com

Received 4 August 2010; Revised 25 November 2010; Accepted 22 December 2010

Academic Editor: R. Pollock

Copyright (C) 2010 Thomas Karsten Kilvaer et al. This is an open access article distributed under the Creative Commons Attribution License, which permits unrestricted use, distribution, and reproduction in any medium, provided the original work is properly cited.

Background. Optimal treatment of nongastrointestinal stromal tumor soft-tissue sarcomas (non-GIST STSs) is resection with wide margins. This study investigates the prognostic impact of the angiogenesis-associated platelet-derived growth factors (PDGFs) and their receptors (PDGFRs) in non-GIST STS patients with wide and nonwide resection margins. Method. Tumor samples and clinical data from 249 patients with non-GIST STS were obtained, and tissue microarrays were constructed for each specimen. Immunohistochemistry was used to evaluate the expression of PDGF-A, - B, - C, and -D and PDGFR- $\alpha$ and $-\beta$. Results. In the multivariate analysis of patients with wide resection margins, high expression of PDGF-B $(P=.013, \mathrm{HR}=2.954$, and $95 \% \mathrm{CI}=$ 1.255-6.956) and the coexpression of PDGF-B and PDGFR- $\alpha$ (overall; $P=.016$, high-low/low-high; $P=.051, \mathrm{HR}=2.678,95 \%$ $\mathrm{CI}=0.996-7.200$, high/high; $P=.004, \mathrm{HR}=3.930,95 \% \mathrm{CI}=1.542-10.015)$ were independent negative prognostic markers for disease-specific survival. Conclusion. PDGF-B and the coexpression of PDGF-B and PDGFR- $\alpha$ are strong and independent prognostic factors in non-GIST STSs with wide resection margins.

\section{Introduction}

Soft-tissue sarcomas (STSs) are mesenchymal-derived tumors comprising about $0.5 \%$ of the annual cancer incidence with an estimate of ten thousand new patients and nearly four thousand related deaths in the USA in 2009 [1]. The STS group consists of more than 50 histological entities [2]. Because of the low incidence and similar ancestry of these tumors it is convenient to group them together when conducting studies $[2,3]$. A proposed way to group these tumors is Ewing family tumors, gastrointestinal stromal tumors (GISTs), and non-GIST STSs, with the latter group consisting of the remaining tumors [3]. Despite improvements in therapy over the last decades the diseasespecific survival (DSS) and progression-free survival (PFS) of sarcoma patients are still poor. The main treatment is resection with wide margins, while radiotherapy is often used in high-grade tumors with both marginal and wide resection margins $[4,5]$. Even when wide resection margins are obtained, a relatively high proportion of patients die $[6,7]$. Several adjuvant chemotherapy-regimes are used for the treatment of sarcomas, but with the exception of childhood rhabdomyosarcomas, Ewing family tumors and GISTs, studies are inconclusive on the effects of these agents $[4,8,9]$. Identification of the subgroup of patients, with wide resection margins and low survival, could prove important, as these patients might benefit from adjuvant therapy.

The platelet-derived growth factor (PDGF) group of signaling molecules consists of four proteins forming five possible ligands in vivo, namely PDGF-AA, PDGF-AB, PDGF-BB, 
PDGF-CC, and PDGF-DD [10]. The platelet-derived growth factor receptors (PDGFRs) are structurally related tyrosinekinase receptors consisting of either $\alpha$ - or $\beta$-chains forming three possible receptors: PDGFR- $\alpha \alpha$, PDGFR- $\alpha \beta$, and PDGFR- $\beta \beta$. The PDGF-AA binds exclusively to the PDGFR$\alpha \alpha$, while PDGF-AB and -CC bind to both the PDGFR- $\alpha \alpha$ and PDGFR- $\alpha \beta$; PDGF-DD binds both PDGFR- $\alpha \beta$ and PDGFR- $\beta \beta$, while PDGF-BB binds all PDGFRs [10].

PDGFs and PDGFRs play a major role in angiogenesis, the recruitment and regulation of tumor stroma, and the regulation of tumor interstitial fluid pressure (IFP) [11]. In addition PDGFs have been shown to function as powerful transforming growth factors, leading cells to progress through cell-cycle and avoid apoptosis [12].

The PDGF/PDGFR pathways have previously been implicated in several sarcomas including GIST, dermatofibrosarcoma protuberans, childhood rhabdomyosarcoma, Kaposis sarcoma, osteosarcoma, and Ewing family sarcoma [11, 13-19]. Regarding non-GIST STSs, there are no conclusive studies on the prognostic impact of PDGF/PDGFRs. Knowledge about the expression and prognostic impact of PDGFs and PDGFRs may be important in identifying patients with wide resection margins and low DSS. With the emerging class of selective small molecule inhibitors targeting these pathways, this might be particularly important. The study presented herein investigates the prognostic impact of PDGFs and PDGFRs in non-GIST STS with wide and nonwide resection margins.

\section{Patients and Methods}

2.1. Patients and Clinical Samples. Primary tumor tissue from anonymized patients diagnosed with non-GIST STS at the University Hospital of North Norway and the hospitals of Arkhangelsk county, Russia, from 1973 through 2006, were collected. In total, 496 patients were registered from the hospital databases. Of these 247 patients were excluded from the study because of missing clinical data $(n=86)$ or inadequate paraffin-embedded fixed tissue blocks $(n=161)$. Thus, 249 patients with complete medical records and adequate paraffin-embedded tissue blocks were eligible.

This report includes followup data as of September 2009. The median followup was 37.6 (range 0.1-391.7) months. Complete demographic and clinical data were collected retrospectively. Formalin-fixed and paraffin-embedded tumor specimens were obtained from the archives of the Departments of Pathology at the University Hospital of North Norway and the hospitals of Arkhangelsk county, Russia. The tumors were graded according to the French Fédération Nationale des Centres de Lutte Contre le Cancer (FNCLCC) system and histologically subtyped according to the World Health Organization guidelines $[2,20]$. Wide resection margins were defined as wide local resection with free microscopic margins or amputation of the affected limb or organ. Nonwide resection margins were defined as marginal or intralesional resection margins, or no surgery.

2.2. Microarray Construction. All sarcomas were histologically reviewed by two trained pathologists (S. Sorbye and
A. Valkov), and the most representative areas of tumor cells (neoplastic mesenchymal cells) were carefully selected and marked on the hematoxylin and eosin (H/E) slide and sampled for the tissue microarray (TMA) blocks. The TMAs were assembled using a tissue-arraying instrument (Beecher Instruments, Silver Springs, MD, USA). The detailed methodology has been previously reported in [21]. Briefly, we used a $0.6 \mathrm{~mm}$ diameter stylet, and the study specimens were routinely sampled with four replicate core samples from different areas of neoplastic tissue. Normal tissue from the patients was used as staining control.

To include all core samples, 12 TMA blocks were constructed. Multiple 5- $\mu \mathrm{m}$ sections were cut with a Micron microtome (HM355S) and stained by specific antibodies for immunohistochemistry (IHC) analysis.

2.3. Immunohistochemistry. The applied antibodies were subjected to in-house validation by the manufacturer for IHC analysis on paraffin-embedded material. The antibodies used in the study were as follows: PDGF-AA (goat polyclonal; AB-221-NA; R\&D Systems; 1:200), PDGF-AB/BB (rabbit polyclonal; RB-9257; Neomarkers; 1:15), PDGF-CC (goat polyclonal; GT15151; Neuromics; 1:80), PDGF-DD (goat polyclonal; AF1159; R\&D Systems; $1: 400$ ), PDGFR- $\alpha$ (rabbit polyclonal; RB-9027; Neomarkers; 1:75), and PDGFR- $\beta$ (rabbit polyclonal; RB-9032; Neomarkers; $1: 25$ ).

Sections were deparaffinized with xylene and rehydrated with ethanol. Antigen retrieval of PDGF-A, -B, -C, and -D was performed by placing the specimen in $0.01 \mathrm{M}$ citrate buffer at $\mathrm{pH} 6.0$ and exposing them to repeated $(\times 2)$ microwave heating of $10 \mathrm{~min}$ at $450 \mathrm{~W}$. PDGF-A, $-B$, and $-C$ were stained using peroxydase/DAB (Dako EnVision+System-HRP/DAB). The primary antibodies were incubated for $30 \mathrm{~min}$ in room temperature. PDGF-D was visualized by adding a secondary antibody conjugated with Biotin, followed by an Avidin/Biotin/Peroxydase complex (Vectastein ABC Elite kit from Vector Laboratories). The primary antibody was incubated overnight at $4^{\circ} \mathrm{C}$. Finally, all slides were counterstained with hematoxylin to visualize the nuclei.

The receptors (PDGFR- $\alpha$ and $-\beta$ ) were stained using Ventana BenchMark XT (Ventana Medical Systems Inc.), procedure iView DAB. Antigen retrieval was done in Tris/EDTA buffer at $\mathrm{pH} 8.4$ for $30 \mathrm{~min}$ (PDGFR- $\alpha$ ) or $60 \mathrm{~min}$ $(\mathrm{PDGFR}-\beta)$ at $37^{\circ} \mathrm{C}$. The primary antibodies were incubated for $30 \mathrm{~min}$ in room temperature.

For each antibody, included negative staining controls, all TMA stainings were done in a single experiment.

2.4. Scoring of Immunohistochemistry. The ARIOL imaging system (Genetix, San Jose, CA) was used to scan the slides of antibody staining of the TMAs. The slides were loaded in the automated slide loader (Applied Imaging SL 50), and the specimens were scanned at low resolution $(1.25 \times)$ and high resolution (20x) using the Olympus BX 61 microscope with an automated platform (Prior). Representative and viable tissue sections were scored manually on computer screen semiquantitatively for cytoplasmic staining. The dominant staining intensity was scored as 0 : negative, 1: weak, 


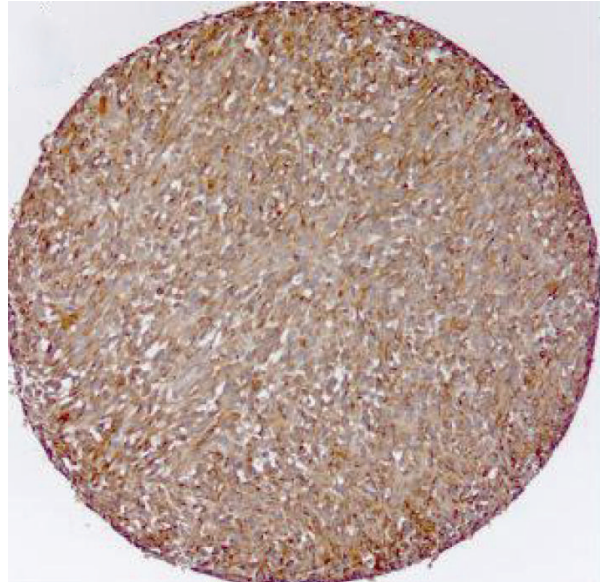

(a)

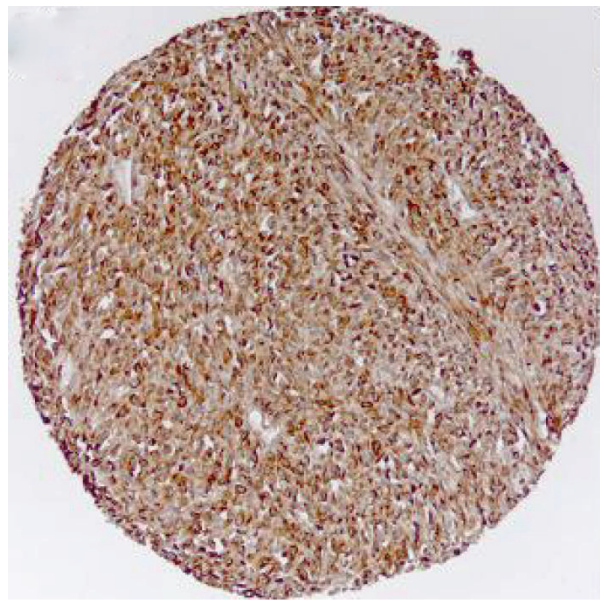

(c)

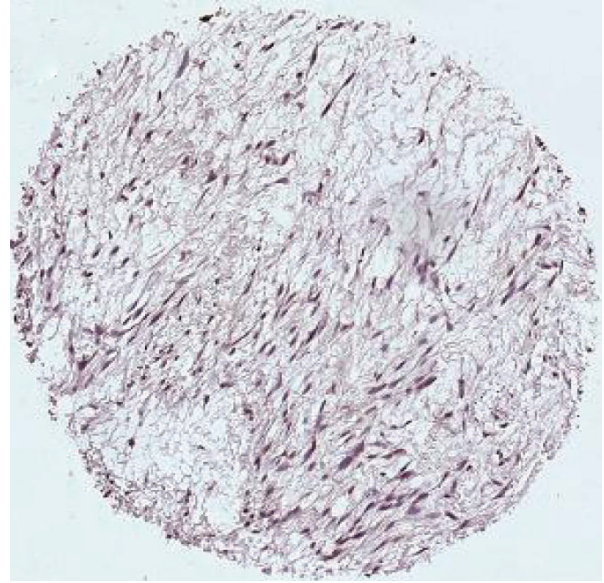

(b)

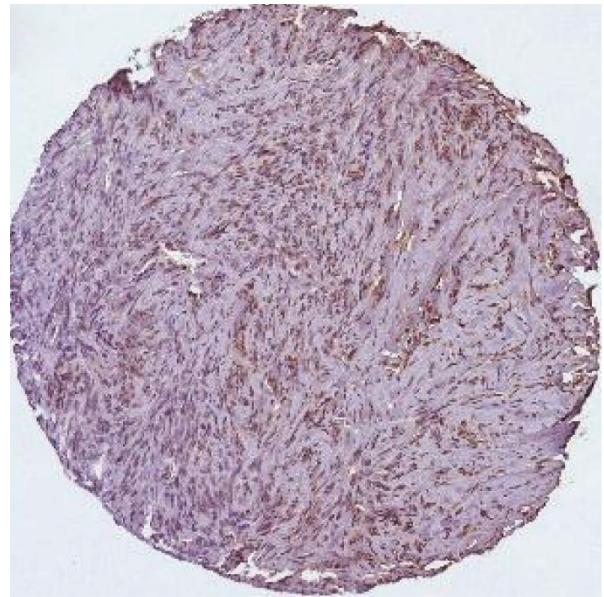

(d)

FIGURE 1: IHC analysis of TMA of nongastrointestinal stromal tumor soft-tissue sarcoma representing different scores for tumor cell PDGFB and PDGFR- $\alpha$. (a) Tumor cell PDGF-B high score; (b) tumor cell PDGF-B low score; (c) tumor cell PDGFR- $\alpha$ high score; (d) tumor cell PDGFR- $\alpha$ low score. Abbreviations: IHC: immunohistochemistry; TMA: tissue microarray; PDGF: platelet-derived growth factor; PDGFR: platelet-derived growth factor receptor.

2: intermediate, and 3: strong. All samples were anonymized and independently scored by two trained pathologists (A. Valkov and S. Sorbye). When assessing a variable for a given core, the observers were blinded to the scores of the other variables and to the outcome. Mean score for duplicate cores from each individual was calculated separately.

High expression in tumor cells was defined as score $\geq 1.5$ (PDGF-A, PDGF-C, and PDGF-B) and $\geq 2$ (PDGF-D, PDGFR- $\alpha$, and PDGFR- $\beta$ ) (Figure 1).

2.5. Statistical Methods. All statistical analyses were done using the statistical package SPSS (Chicago, IL, USA), version 16. The IHC scores from each observer were compared for interobserver reliability by use of a two-way random effect model with absolute agreement definition. The intraclass correlation coefficient (reliability coefficient) was obtained from these results. The Chi-square test and Fishers Exact test were used to examine the association between molecular marker expression and various clinicopathological parameters. Univariate analyses were done using the
Kaplan-Meier method, and statistical significance between survival curves was assessed by the log-rank test. DSS was determined from the date of diagnosis to the time of cancer-related death. To assess the independent value of different pretreatment variables on survival, in the presence of other variables, multivariate analyses were carried out using the Cox proportional hazards model. Only variables of significant value from the univariate analyses were entered into the Cox regression analyses. Probability for stepwise entry and removal was set at.05 and.10, respectively. The significance level used for all statistical tests was $P<.05$.

2.6. Ethical Clearance. The National Data Inspection Board and The Regional Committee for Research Ethics approved the study.

\section{Results}

3.1. Clinopathological Variables. The clinopathological variables are summarized in Table 1. The median age was 59 
TAble 1: Prognostic clinicopathological variables as predictors for disease-specific survival in 249 nongastrointestinal stromal tumor softtissue sarcomas (univariate analyses; log-rank test).

\begin{tabular}{|c|c|c|c|c|c|}
\hline Characteristics & Patients $(n)$ & Patients (\%) & Median survival (months) & 5 -year survival (\%) & $P$ \\
\hline \multicolumn{6}{|l|}{ Age } \\
\hline$\leq 20$ years & 20 & 8 & 15 & 40 & \multirow[t]{3}{*}{.126} \\
\hline $21-60$ years & 113 & 45 & 68 & 52 & \\
\hline$>60$ years & 116 & 47 & 30 & 40 & \\
\hline \multicolumn{6}{|l|}{ Gender } \\
\hline Male & 110 & 44 & 41 & 46 & \multirow[t]{2}{*}{.390} \\
\hline Female & 139 & 56 & 45 & 45 & \\
\hline \multicolumn{6}{|l|}{ Patient nationality } \\
\hline Norwegian & 167 & 67 & 63 & 51 & \multirow[t]{2}{*}{.011} \\
\hline Russian & 82 & 33 & 22 & 34 & \\
\hline \multicolumn{6}{|l|}{ Histological entity } \\
\hline Pleomorphic sarcoma & 58 & 23 & 54 & 47 & \multirow[t]{9}{*}{.001} \\
\hline Leiomyosarcoma & 64 & 26 & 48 & 48 & \\
\hline Liposarcoma & 34 & 14 & NR & 67 & \\
\hline Fibrosarcoma & 20 & 8 & 44 & 50 & \\
\hline Angiosarcoma & 13 & 5 & 10 & 31 & \\
\hline Rhabdomyosarcoma & 16 & 6 & 17 & 38 & \\
\hline MPNST & 11 & 4 & 49 & 45 & \\
\hline Synovial sarcoma & 16 & 6 & 31 & 29 & \\
\hline Sarcoma NOS & 17 & 7 & 9 & 18 & \\
\hline \multicolumn{6}{|l|}{ Tumor localization } \\
\hline Extremities & 89 & 36 & 100 & 53 & \multirow[t]{5}{*}{.348} \\
\hline Trunk & 47 & 29 & 32 & 44 & \\
\hline Retroperitoneum & 37 & 25 & 25 & 38 & \\
\hline Head/neck & 18 & 7 & 15 & 41 & \\
\hline Visceral & 58 & 23 & 30 & 42 & \\
\hline \multicolumn{6}{|l|}{ Tumor size } \\
\hline$\leq 5 \mathrm{~cm}$ & 74 & 30 & 127 & 57 & \multirow[t]{4}{*}{.027} \\
\hline $5-10 \mathrm{~cm}$ & 91 & 37 & 44 & 45 & \\
\hline$>10 \mathrm{~cm}$ & 81 & 32 & 28 & 37 & \\
\hline Missing & 3 & 1 & & & \\
\hline \multicolumn{6}{|l|}{ Malignancy grade } \\
\hline 1 & 61 & 25 & NR & 74 & \multirow{3}{*}{$<.001$} \\
\hline 2 & 98 & 39 & 41 & 45 & \\
\hline 3 & 90 & 36 & 16 & 26 & \\
\hline \multicolumn{6}{|l|}{ Tumor depth } \\
\hline Superficial & 17 & 7 & NR & 93 & \multirow[t]{2}{*}{$<.001$} \\
\hline Deep & 232 & 93 & 36 & 42 & \\
\hline \multicolumn{6}{|l|}{ Metastasis at diagnosis } \\
\hline No & 206 & 83 & 76 & 53 & \multirow[t]{2}{*}{$<.001$} \\
\hline Yes & 43 & 17 & 10 & 10 & \\
\hline \multicolumn{6}{|l|}{ Surgery } \\
\hline Yes & 228 & 91 & 59 & 50 & $<.001$ \\
\hline No & 21 & 9 & 4 & 0 & \\
\hline Resection margins & & & & & \\
\hline Wide & 108 & 43 & NR & 62 & $<.001$ \\
\hline Nonwide/no surgery & 141 & 57 & 21 & 33 & \\
\hline Chemotherapy & & & & & \\
\hline No & 191 & 77 & 52 & 47 & .424 \\
\hline Yes & 58 & 23 & 29 & 40 & \\
\hline Radiotherapy & & & & & \\
\hline No & 176 & 71 & 48 & 46 & .590 \\
\hline Yes & 73 & 29 & 38 & 43 & \\
\hline
\end{tabular}

Abbreviations: NR: not reached; MPNST: malignant peripheral nerve sheath tumor; NOS: not otherwise specified. 
(range 0-91) years, 56\% were female; 167 patients were Norwegian and 82 Russian. The Non-GIST STSs comprised 249 tumors including angiosarcoma $(n=13)$, fibrosarcoma $(n=20)$, leiomyosarcoma $(n=64)$, liposarcoma $(n=34)$, pleomorphic sarcoma $(n=58)$, neurofibrosarcoma/malignant peripheral nerve sheath tumor (MPNST, $n=11)$, rhabdomyosarcoma $(n=16)$, synovial sarcoma ( $n=16)$, and unspecified sarcoma $(n=17)$. The tumor origins were distributed as follows: $36 \%$ extremities, $19 \%$ trunk, 15\% retroperitoneal, $7 \%$ head/neck, and $23 \%$ visceral. Of 228 patients who underwent surgery, 53\% received surgery alone, 24\% surgery and radiotherapy, $18 \%$ surgery and chemotherapy, and 6\% surgery, radiotherapy, and chemotherapy. Besides, 21 patients did not undergo surgery due to inoperable tumor $(n=11)$, high age/other serious diseases $(n=5)$, STS confirmed at autopsy $(n=3)$ and patient refusal $(n=2)$. Of these unresected patients, seven patients received chemotherapy and/or radiotherapy, whereas 14 patients received no anticancer therapy.

3.2. Interobserver Variability. Interobserver scoring agreement was tested for one ligand (PDGF-B) and one receptor $(\mathrm{PDFGR}-\alpha)$. The intraclass correlation coefficients were 0.890 for PDGF-B $(P<.001)$ and 0.892 for PDFGR- $\alpha$ $(P<.001)$ indicating good reproducibility between the two investigating pathologists.

3.3. Expression of PDGFs/PDGFRs and Their Correlations. PDGF/PDGFR-expression was observed in the cytoplasm of tumor cells. No correlation was seen between increased tumor PDGF/PDGFR expression and tumor depth, surgery, radiotherapy, or chemotherapy. Among the most interesting correlations, PDGFR- $\alpha$ correlated with malignancy grade (high expression; grade 1: $25 \%$, grade 2 : $39 \%$, grade 3 : $54 \%, P=.003)$ and PDGFR- $\beta$ was more often expressed in patients with metastasis at diagnosis (35\%, versus no metastasis at diagnosis, $18 \%, P=.015)$.

3.4. Univariate Analyses. Among demographic and clinicopathological variables in the total material, patient nationality $(P=.011)$, histological entity $(P=.001)$, tumor size $(P=.027)$, malignancy grade $(P<.001)$, tumor depth $(P<$ $.001)$, metastasis at diagnosis $(P<.001)$, surgery $(P<.001)$, and resection margins $(P<.001)$ were significant prognostic indicators of DSS (Table 1).

In the subgroup with wide resection margins, patient nationality $(P<.001)$, malignancy grade $(P<.001)$, tumor depth $(P=.009)$, and metastasis at diagnosis $(P<.001)$ were significant prognostic indicators of DSS. In the subgroup with nonwide resection margins, malignancy grade $(P<$ $.001)$, metastasis at diagnosis $(P<.001)$, surgery $(P<.001)$, and histological entity $(P=.004)$ were significant prognostic indicators of DSS.

The influence on DSS by the PDGFs and PDGFRs is shown in Table 2 and Figure 2. In the total material, high expressions of PDGFR- $\alpha(P=.004)$ and PDGFR- $\beta$ $(P=.047)$ were significant negative prognostic indicators of DSS. In the subgroup with wide resection margins, high expressions of PDGF-B $(P=.007)$, PDGF-D $(P=.029)$,
PDGFR- $\alpha(P=.001)$, and PDGFR- $\beta(P=.022)$ were significant negative prognostic indicators of DSS, while in the subgroup with nonwide resection margins none of the PDGFs or PDGFRs were significant indicators of DSS.

3.5. Multivariate Cox Proportional Hazards Analyses. Results of the multivariate analyses are presented in Tables 3 and 4 . In the total material, tumor depth $(P=.019)$, tumor size $(P=.034)$, high malignancy grade $(P<.001)$, lack of surgery $(P<.001)$, nonwide resection margins $(P=.013)$, and metastasis at diagnosis $(P<.001)$ were significant independent prognostic indicators of DSS (Table 3). In the subgroup with wide resection margins, Russian nationality $(P=.012)$, high malignancy grade $(P=.005)$, metastasis at diagnosis $(P=.001)$, and high expression of PDGF-B $(P=$ $.013, \mathrm{HR}=2.954,95 \% \mathrm{CI}=1.255-6.956)$ were significant independent prognostic indicators of DSS (Table 4). In the subgroup with nonwide resection margins, high malignancy grade $(P<.001)$, lack of surgery $(P<.001)$, and metastasis at diagnosis $(P<.001)$ were significant independent prognostic indicators of DSS.

3.6. Coexpression of PDGF-B and PDGFR- $\alpha$. It is pertinent to assess the coexpression between PDGFs and their receptors as these can represent additive and/or synergic effects on DSS. In univariate analyses, the coexpression of PDGF-B and PDGFR- $\alpha$ was a significant negative prognostic indicator of DSS both in the total material $(P=.020)$ and in the subgroup with wide resection margins $(P=.001)$. In the latter group, the coexpression was a significant independent negative prognostic indicator of DSS (overall; $P=.016$, intermediate; $P=.051, \mathrm{HR}=2.678,95 \% \mathrm{CI}=0.996-7.200$, high; $P=.004, \mathrm{HR}=3.930,95 \% \mathrm{CI}=1.542-10.015)$.

\section{Discussion}

The main treatment for non-GIST STSs is surgery with wide resection margins [4]. The 5-year survival is 30\% in the group with nonwide resection margins and 60\% among those with wide resection margins. The explanation for the modest survival, despite wide resection margins, might be micrometastasis into the surrounding tissue, lymphogenic regional spread, or hematogenous metastasis. Any possibility to identify those patients who will subsequently succumb to progression and metastasis from their resected sarcoma within the wide resection margin group will be pivotal, as these patients may benefit from adjuvant therapy.

We present a large-scale retrospective study of the prognostic impact of PDGF-A, -B, -C, and -D and PDGFR- $\alpha$ and $-\beta$ in non-GIST STS patients. High expression of PDGF$\mathrm{B}$ and the coexpression of PDGF-B and PDGFR- $\alpha$ were significant independent negative prognostic indicators of DSS in those with wide resection margins. To our knowledge this is the first evaluation of PDGFs and PDGFRs in nonGIST STSs according to resection margins [22].

The major limitation of this study consists of tumor heterogeneity as different types of non-GIST STS, diverse disease sites, as well as different therapies are included. These points are, at least partly, accounted for by the multivariate 


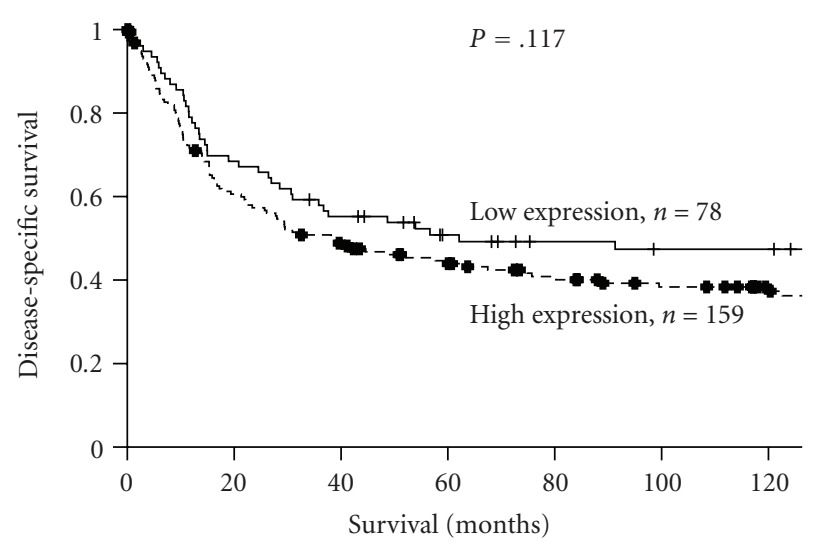

(a) PDGF-B, total material

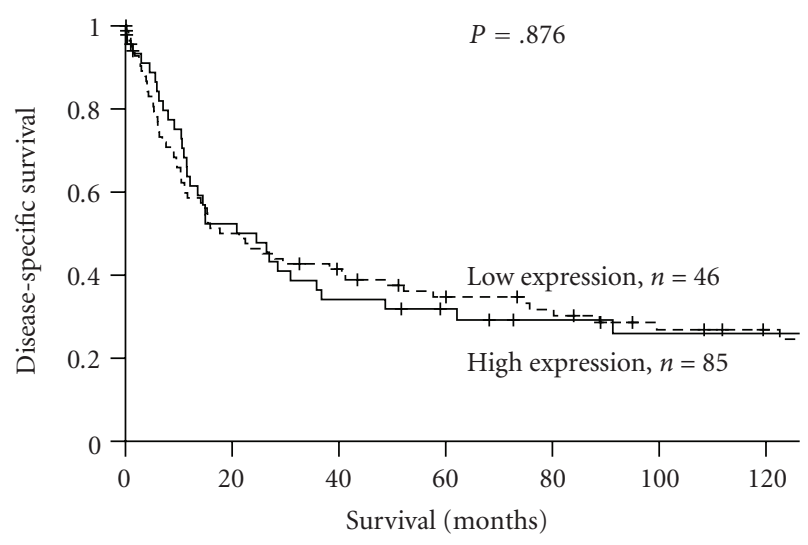

(c) PDGF-B, nowide resection margins

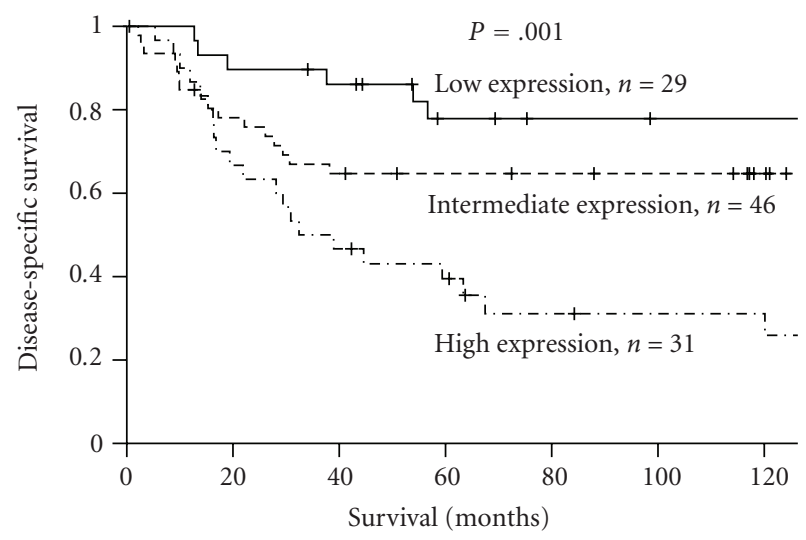

(e) PDGF-B \& PDGFR- $\alpha$, wide resection margins

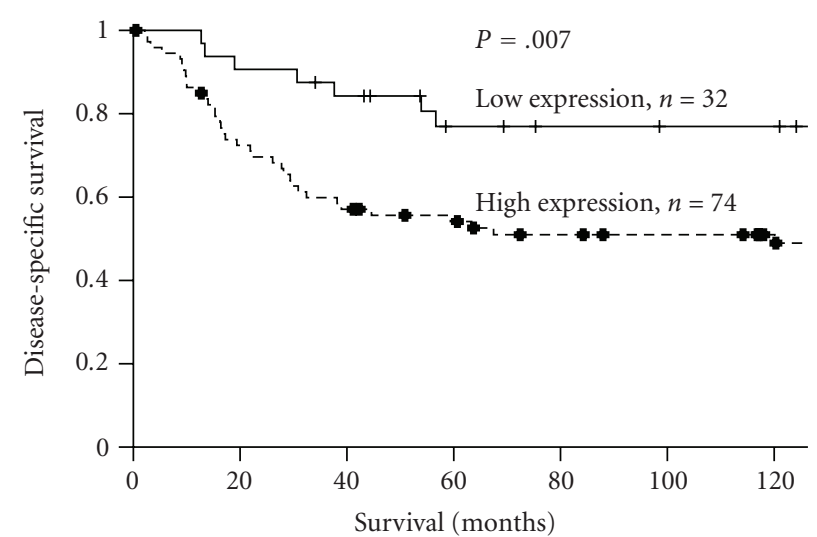

(b) PDGF-B, wide resection margins

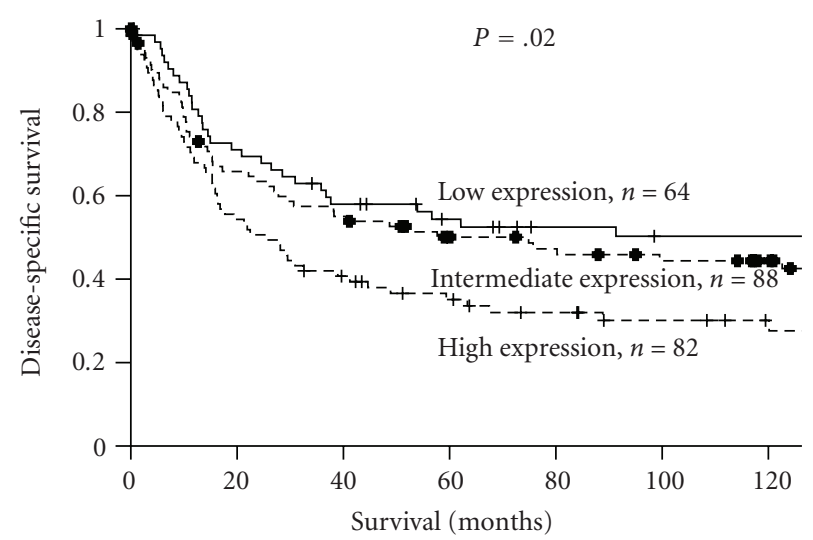

(d) PDGF-B and PDGFR- $\alpha$, total material

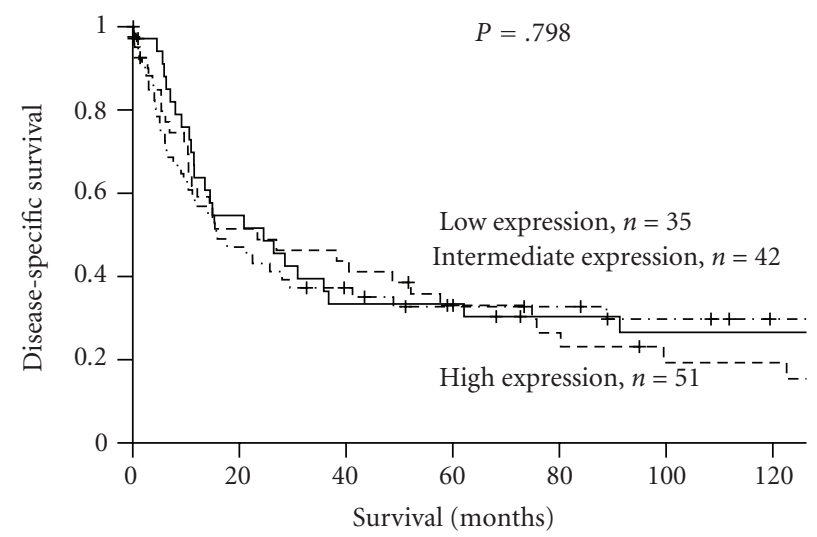

(f) PDGF-B \& PDGFR- $\alpha$, non-wide resection margins

FIGURE 2: Disease-specific survival curves for PDGF-B and the coexpression of PDGF-B \& PDGFR- $\alpha$ in the overall material and in patients with wide and nonwide resection margins. (a) PDGF-B, total material; (b) PDGF-B, wide resection margins; (c) PDGF-B, nonwide resection margins; (d) coexpression of PDGF-B and PDGFR- $\alpha$, total material; (e) coexpression of PDGF-B and PDGFR- $\alpha$, wide resection margins; (f) coexpression of PDGF-B and PDGFR- $\alpha$, nonwide resection margins. Abbreviations: PDGF: platelet-derived growth factor; PDGFR: platelet-derived growth factor receptor. 


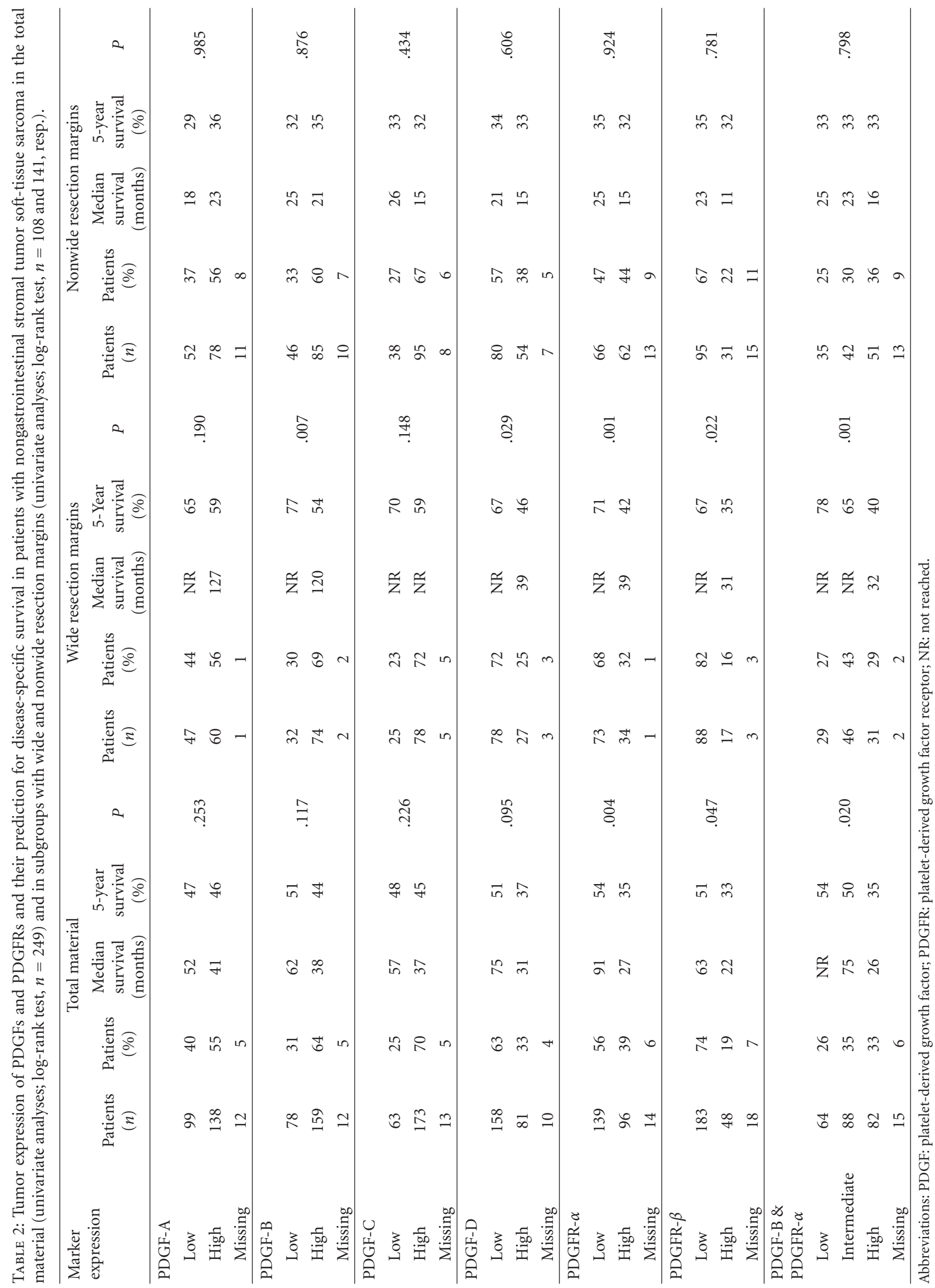


TABLE 3: Result of the Cox regression analysis among all patients.

\begin{tabular}{lccc}
\hline Factor & Hazard ratio & $95 \%$ CI & $P$ \\
\hline $\begin{array}{l}\text { Tumor depth } \\
\quad \text { Superficial }\end{array}$ & 1.000 & & \\
$\quad$ Deep & 9.765 & $1.339-71.189$ & .025 \\
\hline Tumor size & & & $.028^{*}$ \\
$\quad \leq 5 \mathrm{~cm}$ & 1.000 & & \\
$5-10 \mathrm{~cm}$ & 1.379 & $0.855-2.223$ & .187 \\
$>10 \mathrm{~cm}$ & 1.960 & $1.192-3.221$ & .008 \\
\hline Malignancy grade & & & $<.001^{*}$ \\
1 & 1.000 & & \\
2 & 2.914 & $1.605-5.292$ & $<.001$ \\
3 & 4.600 & $2.527-8.373$ & $<.001$ \\
\hline Surgery & & & \\
$\quad$ Yes & 1.000 & & \\
$\quad$ No & 8.628 & $4.269-17.438$ & $<.001$ \\
\hline Resection margins & & & \\
Wide & 1.000 & & \\
Nonwide & 1.847 & $1.243-2.744$ & .003 \\
\hline Metastasis at time of diagnosis & & & \\
No & 1.000 & & \\
Yes & 2.562 & $1.644-3.991$ & $<.001$ \\
\hline * & & &
\end{tabular}

${ }^{*}$ Overall significance as a prognostic factor.

analysis. Recent studies have suggested new ways of grouping STSs according to their mutational status in addition to histology [22]. A homologous population with knowledge of mutational status of common sarcoma mutations would have been interesting, but this is difficult to arrange in the setting of a retrospective study.

The major strengths of this study lie in the size of the cohort, the consistency of the clinical variables with previous studies on sarcomas, and the biological soundness of our findings.

In 1994, Wang et al. reported a positive correlation between increased PDGF-B expression and high histological malignancy grade of STSs using a set of 56 STSs of all grades including benign tumors [23]. Herein, we did not observe a correlation between PDGF-B expression and histological grade, but PDGF-B appeared to be a significant independent negative prognostic marker for DSS in patients with wide resection margins.

Experiments in mice have shown involvement of endothelial-derived PDGF-B, in both pericyte and vascular smooth muscle cell (VSM) recruitment and stabilization, during blood vessel formation [24-26]. Pericytes have been implicated in differentiation and stabilization of blood vessels, and VSMs are an important component of blood vessel walls. As adequate blood supply is pivotal in tumor formation, tumor-derived PDGF-B could represent a way for recruitment of pericytes and VSMs, leading to tumor growth and increased viability and tumor forming capabilities of metastatic tumors. High tumor interstitial fluid pressure (IFP) has been shown to lower chemotherapeutic response due to lower transcapillary transport of chemotherapeutic
TABLE 4: Results of the Cox regression analysis among patients with wide resection margins.

\begin{tabular}{lccc}
\hline Factor & Hazard ratio & $95 \%$ CI & $P$ \\
\hline $\begin{array}{l}\text { Patient nationality } \\
\quad \text { Norwegian }\end{array}$ & 1.000 & & \\
$\quad$ Russian & 2.292 & $1.199-4.383$ & .012 \\
\hline $\begin{array}{l}\text { Malignancy grade } \\
1\end{array}$ & 1.000 & & $.005^{*}$ \\
2 & 4.438 & $1.262-15.609$ & .020 \\
3 & 7.368 & $2.138-25.389$ & .002 \\
\hline Metastasis at time of diagnosis & & & \\
$\quad$ No & 1.000 & & \\
$\quad$ Yes & 3.939 & $1.801-8.613$ & .001 \\
\hline PDGF-B & & & \\
$\quad$ Low & 1.000 & & \\
$\quad$ High & 2.954 & $1.255-6.956$ & .013 \\
\hline * Overall significance as a prognostic factor. Abbreviations: PDGF: platelet- \\
derived growth factor.
\end{tabular}

agents [27]. PDGF-B has been shown to increase IFP and may play a role in tumors showing unexpected poor response to chemotherapy [11]. Studies in mice demonstrated that blocking PDGFs and PDGFRs resulted in better response to chemotherapy in several experimental tumor types [28,29]. These findings indicate that increased expression of PDGFs and PDGFRs might perturb the effect of chemotherapy and therefore contribute to explain why the response to chemotherapy remains controversial in non-GIST STSs. PDGF-B primarily signals through PDGFR- $\beta$ but has also been shown to signal through PDGFR- $\alpha$ [10]. Upon receptor activation, PDGF-B has strong transforming capabilities, activating several intracellular pathways, including PI3K, PLC $\gamma$, SRC, and RAS. Activation of these pathways might lead to increased cell cycling and avoidance of apoptosis [12]. In univariate analysis, high expression of PDGF-D was a significant negative prognostic marker in non-GIST STSs with wide resection margins. PDGF-D has previously been shown to exhibit extensive transforming and angiogenic abilities [30]. Vessels formed in PDGF-D-driven tumors show great similarity to vessels formed by PDGF-B-driven tumors, suggesting that PDGF-D, in absence of PDGF-B, can take over some or all of PDGF-Bs angiogenic functions [30]. Further, both PDGFR- $\alpha$ and $-\beta$ were significant negative prognostic markers for non-GIST STSs with wide resection margins. PDGFRs have been regarded as a "driving force" in many human cancers, including GISTs, through autoactivation and constitutive signaling [19]. The fact that PDGFR$\alpha$ correlated with malignancy grade and that PDGFR- $\beta$ correlated with metastasis at diagnosis suggests that this might also be the case in non-GIST STSs, although further investigation is warranted.

We also found the coexpression of PDGF-B and PDGFR- $\alpha$ to have a significant independent negative prognostic impact in non-GIST STS with wide resection margins. A significantly lower survival in the high-high group versus the low-low and the high-low/low-high groups suggests 
an additive or possibly synergic effect (Figure 2). The exact mechanism for this finding is not clear, but there are several possible explanations. As previously mentioned, PDGF-B preferably signals through PDGFR- $\beta$ but can also signal through PDGFR- $\alpha$. When both PDGFRs and PDGF-B are expressed in the tumor it is likely that both pathways are simultaneously stimulated by PDGF-B or by consecutive stimulation of the PDGFR- $\beta$ with constitutively active PDGFR- $\alpha$. Part of the effect might also come from PDGF$B$ interactions with stromal components like pericytes and VSMs. Simultaneous stimulation of both PDGFR axes, together with the proposed angiogenic and stromal regulatory effects of PDGF-B, may explain the decreased DSS in non-GIST STS patients with wide resection margins.

Small-molecule inhibitors of tyrosine kinase receptors have been used in the treatment of GISTs, dermatofibrosarcoma protuberans, chronic myelogenous leukemia, and others, demonstrating great therapeutic potential [31]. Cell line experiments and animal studies suggest synergistic effects and reduced side effects of these inhibitory agents together with conventional chemo- or radiotherapy $[28,29]$.

\section{Conclusion}

Despite wide resection margins, one third of patients still die of non-GIST STS. We have identified high expression of PDGF-B to be an independent negative prognostic factor for DSS in non-GIST STS patients with wide resection margins. Further, PDGF-B and PDGFR- $\alpha$ coexpression revealed a wide resection margin subgroup with particularly poor DSS. Our results indicate involvement of PDGF and/or PDGFRs in non-GIST STS pathogenesis. The mechanisms responsible for this involvement have to be further elucidated and finally validated in prospective clinical trials. With the evolving small-molecule inhibitors targeting these pathways, PDGFs and PDGFRs may become important targets in the treatment of non-GIST STSs.

\section{Funding}

This study was funded by the Northern Norway Health Authority, The Norwegian Childhood Cancer Network, The Norwegian Sarcoma Group, and The Norwegian Cancer Society. The funding sources had no influence on the study design, data collection, analysis, and interpretation of data, in the writing of the paper, or in the decision to submit the paper for publication.

\section{Conflict of Interests}

The authors declare that they have no conflict of interests.

\section{Acknowledgments}

Thanks are due to Frode Skjold for coupling of databases, Magnus L. Persson for making the TMA blocks, and Helge Stalsberg for gathering of clinical information.

\section{References}

[1] A. Jemal, R. Siegel, E. Ward, Y. Hao, J. Xu, and M. J. Thun, "Cancer statistics, 2009," CA Cancer Journal for Clinicians, vol. 59, no. 4, pp. 225-249, 2009.

[2] C. D. M. Fletcher, K. K. Unni, and F. Mertens, Pathology and Genetics of Tumours of Soft Tissue and Bone, IARC Press, Lyon, France, 2002.

[3] S. Sleijfer, W. T. A. Van Der Graaf, and J. Y. Blay, "Angiogenesis inhibition in non-GIST soft tissue sarcomas," Oncologist, vol. 13, no. 11, pp. 1193-1200, 2008.

[4] W. M. Mendenhall, D. J. Indelicato, M. T. Scarborough et al., "The management of adult soft tissue sarcomas," American Journal of Clinical Oncology, vol. 32, no. 4, pp. 436-442, 2009.

[5] A. Kaushal and D. Citrin, "The role of radiation therapy in the management of sarcomas," Surgical Clinics of North America, vol. 88, no. 3, pp. 629-646, 2008.

[6] I. C. Dickinson, D. J. Whitwell, D. Battistuta et al., "Surgical margin and its influence on survival in soft tissue sarcoma," ANZ Journal of Surgery, vol. 76, no. 3, pp. 104-109, 2006.

[7] J. J. Lewis, D. Leung, J. M. Woodruff, and M. F. Brennan, "Retroperitoneal soft-tissue sarcoma: analysis of 500 patients treated and followed at a single institution," Annals of Surgery, vol. 228, no. 3, pp. 355-365, 1998.

[8] K. Thornton, "Chemotherapeutic management of soft tissue sarcoma," Surgical Clinics of North America, vol. 88, no. 3, pp. 647-660, 2008.

[9] D. M. Loeb, K. Thornton, and O. Shokek, "Pediatric soft tissue sarcomas," Surgical Clinics of North America, vol. 88, no. 3, pp. 615-627, 2008.

[10] L. Fredriksson, H. Li, and U. Eriksson, "The PDGF family: four gene products form five dimeric isoforms," Cytokine and Growth Factor Reviews, vol. 15, no. 4, pp. 197-204, 2004.

[11] A. Östman, "PDGF receptors-mediators of autocrine tumor growth and regulators of tumor vasculature and stroma," Cytokine and Growth Factor Reviews, vol. 15, no. 4, pp. 275286, 2004.

[12] M. Tallquist and A. Kazlauskas, "PDGF signaling in cells and mice," Cytokine and Growth Factor Reviews, vol. 15, no. 4, pp. 205-213, 2004.

[13] P. M. Armistead, J. Salganick, J. S. Roh et al., "Expression of receptor tyrosine kinases and apoptotic molecules in rhabdomyosarcoma: correlation with overall survival in 105 patients," Cancer, vol. 110, no. 10, pp. 2293-2303, 2007.

[14] G. McArthur, "Dermatofibrosarcoma protuberans: recent clinical progress," Annals of Surgical Oncology, vol. 14, no. 10, pp. 2876-2886, 2007.

[15] H. B. Koon, G. J. Bubley, L. Pantanowitz et al., "Imatinibinduced regression of AIDS-related Kaposi's sarcoma," Journal of Clinical Oncology, vol. 23, no. 5, pp. 982-989, 2005.

[16] A. Shimizu, K. P. O’Brien, T. Sjöblom et al., “The dermatofibrosarcoma protuberans-associated collagen type $\mathrm{I} \alpha 1 /$ platelet-derived growth factor (PDGF) B-chain fusion gene generates a transforming protein that is processed to functional PDGF-BB," Cancer Research, vol. 59, no. 15, pp. 3719-3723, 1999.

[17] M. T. Hueman and R. D. Schulick, "Management of gastrointestinal stromal tumors," Surgical Clinics of North America, vol. 88, no. 3, pp. 599-614, 2008.

[18] M. C. Blandford, F. G. Barr, J. C. Lynch, R. L. Randall, S. J. Qualman, and C. Keller, "Rhabdomyosarcomas utilize developmental, myogenic growth factors for disease advantage: a report from the children's oncology group," Pediatric Blood and Cancer, vol. 46, no. 3, pp. 329-338, 2006. 
[19] C. Y. Du, Y. Q. Shi, YE. Zhou, H. Fu, and G. Zhao, "The analysis of status and clinical implication of KIT and PDGFRA mutations in gastrointestinal stromal tumor (GIST)," Journal of Surgical Oncology, vol. 98, no. 3, pp. 175-178, 2008.

[20] L. Guillou, J. M. Coindre, F. Bonichon et al., "Comparative study of the National Cancer Institute and French Federation of Cancer Centers Sarcoma Group grading systems in a population of 410 adult patients with soft tissue sarcoma," Journal of Clinical Oncology, vol. 15, no. 1, pp. 350-362, 1997.

[21] T. Donnem, S. Al-Saad, K. Al-Shibli et al., "Inverse prognostic impact of angiogenic marker expression in tumor cells versus stromal cells in non-small cell lung cancer," Clinical Cancer Research, vol. 13, no. 22, pp. 6649-6657, 2007.

[22] E. Wardelmann, H.-U. Schildhaus, S. Merkelbach-Bruse et al., "Soft tissue sarcoma: from molecular diagnosis to selection of treatment. Pathological diagnosis of soft tissue sarcoma amid molecular biology and targeted therapies," Annals of Oncology, vol. 21, supplement 7, pp. vii265-vii269, 2010.

[23] J. Wang, M. D. Coltrera, and A. M. Gown, "Cell proliferation in human soft tissue tumors correlates with platelet- derived growth factor B chain expression: an immunohistochemical and in situ hybridization study," Cancer Research, vol. 54, no. 2, pp. 560-564, 1994.

[24] A. Abramsson, P. Lindblom, and C. Betsholtz, "Endothelial and nonendothelial sources of PDGF-B regulate pericyte recruitment and influence vascular pattern formation in tumors," Journal of Clinical Investigation, vol. 112, no. 8, pp. 1142-1151, 2003.

[25] C. Betsholtz, "Insight into the physiological functions of PDGF through genetic studies in mice," Cytokine and Growth Factor Reviews, vol. 15, no. 4, pp. 215-228, 2004.

[26] P. Lindblom, H. Gerhardt, S. Liebner et al., "Endothelial PDGF-B retention is required for proper investment of pericytes in the microvessel wall," Genes and Development, vol. 17, no. 15, pp. 1835-1840, 2003.

[27] C. H. Heldin, K. Rubin, K. Pietras, and A. Östman, "High interstitial fluid pressure-an obstacle in cancer therapy," Nature Reviews Cancer, vol. 4, no. 10, pp. 806-813, 2004.

[28] K. Pietras, K. Rubin, T. Sjöblom et al., "Inhibition of PDGF receptor signaling in tumor stroma enhances antitumor effect of chemotherapy," Cancer Research, vol. 62, no. 19, pp. 5476$5484,2002$.

[29] K. Pietras, M. Stumm, M. Hubert et al., "STI571 enhances the therapeutic index of epothilone B by a tumor-selective increase of drug uptake," Clinical Cancer Research, vol. 9, no. 10 I, pp. 3779-3787, 2003.

[30] H. Li, L. Fredriksson, X. Li, and U. Eriksson, "PDGF-D is a potent transforming and angiogenic growth factor," Oncogene, vol. 22, no. 10, pp. 1501-1510, 2003.

[31] J. Baselga, "Targeting tyrosine kinases in cancer: the second wave," Science, vol. 312, no. 5777, pp. 1175-1178, 2006. 


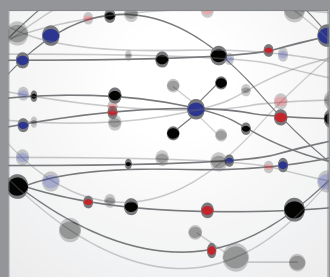

The Scientific World Journal
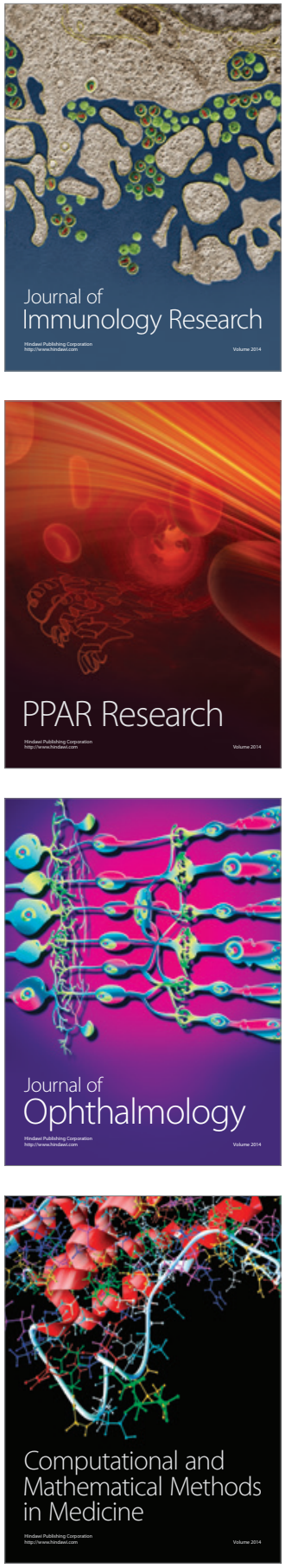

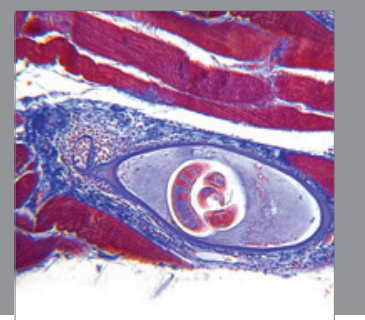

Gastroenterology

Research and Practice
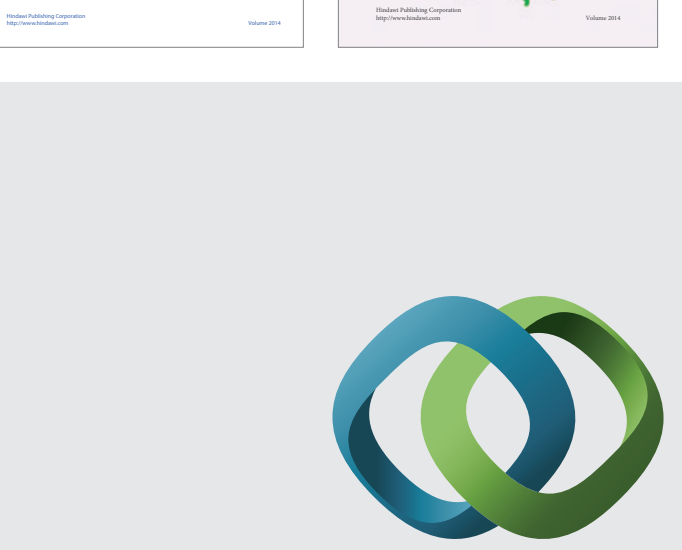

\section{Hindawi}

Submit your manuscripts at

http://www.hindawi.com
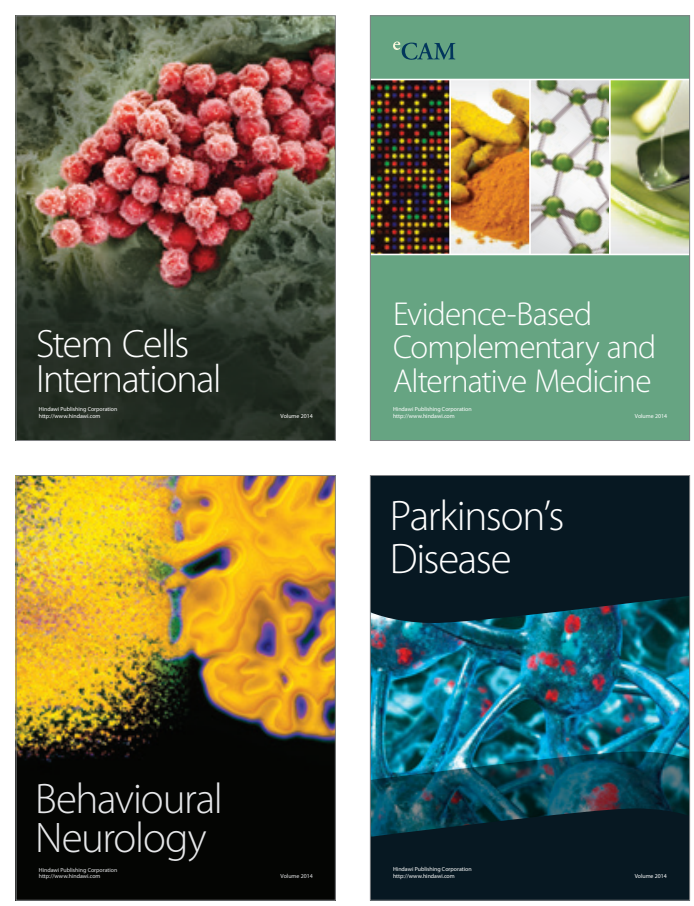

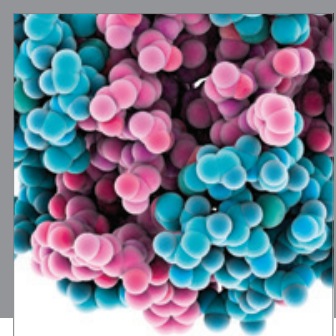

Journal of
Diabetes Research

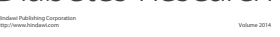

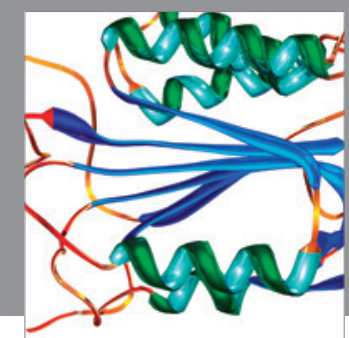

Disease Markers
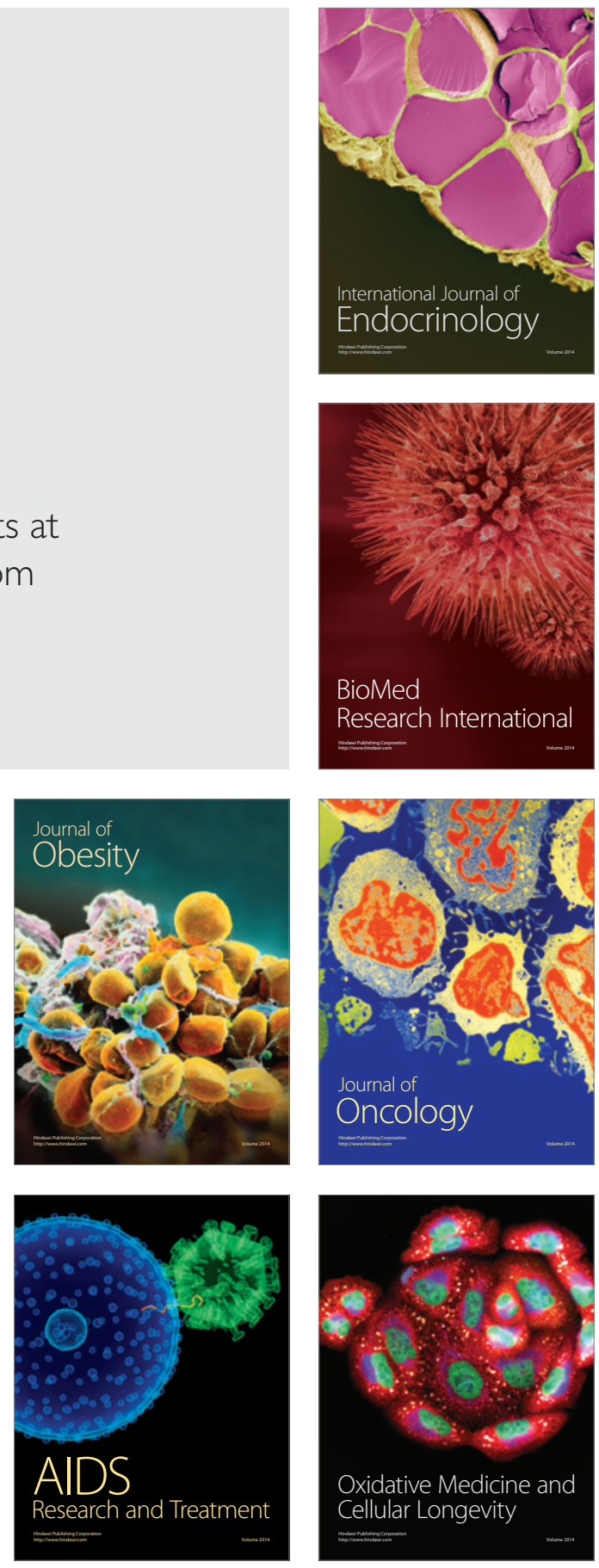